\title{
Common-mode noise filtering with space-divided differential $2 \times 2$ VLC for V2V applications
}

\author{
Balázs Matolcsy ${ }^{1}$ D $\cdot$ Eszter Udvary $^{1} \cdot$ Ágoston Schranz $^{1}$
}

Received: 12 August 2020 / Accepted: 7 March 2021 / Published online: 26 March 2021

(c) The Author(s) 2021

\begin{abstract}
Vehicle-to-Vehicle (V2V) Communication is one of the most promising application opportunities of Visible Light Communication (VLC). This paper suggests a novel transmission technique for VLC-V2V applications, called 2x2 Differential Transmission (2x2 DT). This transmission method exhibits excellent common-mode optical noise rejection performance. This beneficiary property is presented first with simulation results and later with real-life urban traffic measurement as well. After the promising simulation results, a demonstrational automotive-grade VLC-V2V link is presented and investigated for evaluation purposes. The link does not contain electrical filters, optical filters nor optical lenses, hence link costs are kept low and versatility is preserved. With our proposed $2 \times 2$ differential solution, both the transmission quality and common-mode optical noise rejection performance is outstanding. Due to the increased sensitivity of the differential VLC transmission regarding crosstalk between channels, a method is suggested for crosstalk reduction for high-quality differential transmission.
\end{abstract}

Keywords VLC $\cdot$ V2V $\cdot$ Differential VLC $\cdot$ Noise reduction · VLC MIMO · Noise impacts

\section{Introduction}

In the last few decades, there has been an exponential growth regarding high-efficiency semiconductor-based LED (Light Emitting Diode) lighting equipment. This trend spread the ability to implement visible light communication (VLC) systems in various areas Adiono, T., et al. 2016. One of the most promising applications of VLC in the last ten years has been Vehicle-to-Vehicle (V2V) communication (Ferraz and Santos 2015; Mohammed et al. 2016). VLC-V2V has gained substantial attention, as the autonomous, connected vehicles concept emerged during the last decade. As modern

Balázs Matolcsy

matolcsy.balazs@vik.bme.hu

Eszter Udvary

udvary.eszter@vik.bme.hu

Ágoston Schranz

schranz.agoston@vik.bme.hu

1 Budapest University of Technology and Economics, Budapest, Hungary 
vehicles are tending to use LED headlights (Jong-Ho et al. 2013; Uysal 2015) and LED taillights, this provides a range of automotive VLC-V2V opportunities (Kim et al. 2014; Siddiqi et al. 2016). As the transmission channel has time-variant properties and greatly depends on weather and visibility conditions Chen et al. (2016), there are many studies available where the VLC-V2V channel is modeled (Uysal 2015; Alsalami et al. 2019; Eldeeb et al. 2019; Karbalayghareh et al. 2020; Lee et al. 2012). There are also studies on the specific impact of some dedicated weather conditions (e.g. fog and rain) (Elamassie et al. 2018; Eso et al. 2019; Kim et al. 2015) to the VLC-V2V channel. As VLC-V2V is an alternative to short-range radiofrequency (RF) communication, the VLC channel is compared to the RF-V2V channel in Cheng (2018). The VLC-V2V channel is similar to the short-range Free-Space-Optical (FSO) channel with the addition of potentially larger time-variance, due to the movement of the transceiver endpoints. In most cases, a Line-of-Sight (LOS) transmission is assumed. There are also studies on non-LOS cases, discussed in Tebruegge et al. (2019), with an amount of attenuation caused by weather and other environmental effects (Karbalayghareh et al. 2020; Eso et al. 2019; Singh et al. 2019). The VLC-V2V channel is substantially affected by natural ambient light, sun irradiance Islim et al. (2018) and artificial light sources: traffic lights, public lighting, other VLC interference from nearby vehicles or objects (Beshr et al. 2015; Chung and Oh 2013). These noises have a significant impact on the quality of the VLC-V2V link. Therefore, their effects must be taken into consideration.

Signal, noise and interference on the receiver side $(R X)$ In VLC-V2V links the majority of optical receivers are either high-speed cameras (Eso et al. 2019; Halawi 2019; Takai 2014) or photodiode based amplified detectors (Chung and Oh 2013; Ahmed and Bermak 2019). In several experimental VLC-V2V links, only one receiver is attached to the back or the front section of the vehicle. This single receiver structure collects the utile transmitter (TX), noise, and interference signals. The signal from noise and interference separation can be implemented in both the optical and electrical domains. Optical domain separation can be performed by using polarization or wavelength filters (Chung and Oh 2013; Tebruegge et al. 2019). However, both polarization and wavelength filters have a considerable amount of insertion loss. Electrical domain separation is carried out with analog or digital bandpass filters (Ahmed and Bermak 2019; Adiono et al. 2016; Chang et al. 2017). However, if there are multiple time-varying noise or interference sources present, their real-time tracking and suppression would be remarkably difficult. If the noise or interference source's signal overlaps with the utile signal in the frequency domain, filtering is useless. There have been several approaches published recently regarding noise filtering in VLC-V2V systems. However, it is not easy to find a proper comparison base, as most VLC-V2V systems are custom-built. In order to place our solution among the several presented approaches, some of the recently published solutions are listed in Table 1. Our aimed solution is shown in the last row.

The aim of this paper is to introduce a new method for reducing the effects of commonmode optical noise in VLC-V2V applications, without expensive optical wavelength filters, polarization filters, electrical domain filters, FPGAs (Field-Programmable-Gate-Array) thus providing a cost-efficient alternative to other known solutions, presented in Table 1. The rest of the paper is organized as follows: in Sect. 1 the differential VLC-V2V concept is introduced, then in Sect. 2 the preliminary simulation results and mathematical background are presented. In Sect. 3 the real-world noise measurement results are shown, afterwards the last section discusses our measurement results with the proposed differential VLC-V2V design in presence of balanced common-mode noise. Our proposed setup is compared with the conventional VLC-V2V solution. In the end, the paper is concluded. 


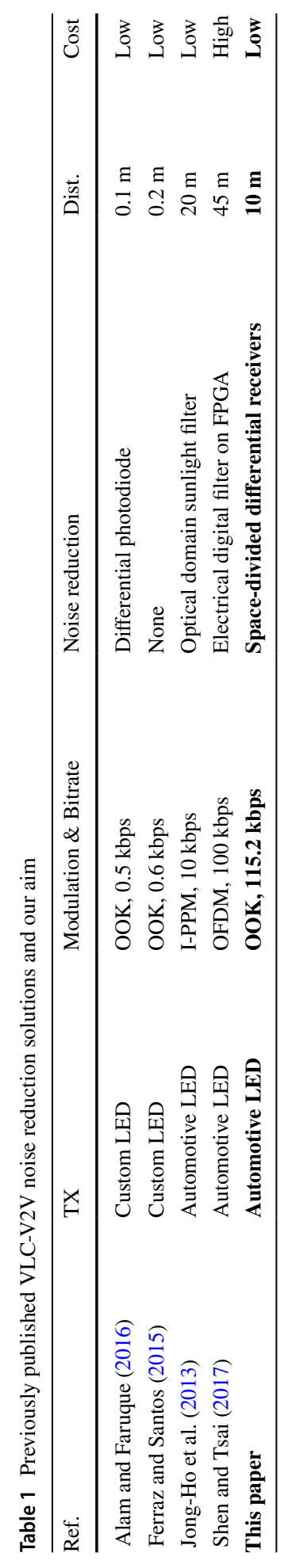


The $2 \times 2$ Differential VLC-V2V Concept As investigated in several studies, the VLC$\mathrm{V} 2 \mathrm{~V}$ channel is loaded with a multitude of optical noises (traffic lights, public lighting etc.). The question is whether there is a way to eliminate the need for optical and electrical filters and reduce optical noises in VLC-V2V systems at the same time.

The idea of introducing two identical VLC receivers separated in space and simultaneously subtracting their signals is the key to differential VLC-V2V. If the received noise time-functions contain components on each receiver that are proportional to each other, these components are considered common-mode noise. In special situations, where the proportion is exactly 1 , the noise is considered as balanced common-mode noise. If the receiver signals are subtracted from each other, common-mode noise is reduced. The reduction efficiency depends on the common-mode noise balance measured on the two receivers. However, the differentially operated receivers require differential transmitters in order to avoid utile signal loss due to the subtraction operation. In VLC-V2V systems this differential transmission can be realized by operating transmitters in a complementary manner. Furthermore, as the differentially operated receivers reduce common-mode signals, the transmitted differential VLC signals should be separated in space and focused only on the designated receiver (TX1 to RX1 only and TX2 to RX2 respectively). Crosstalk between transmitted signals may cause serious performance decay at the receivers.

Let this solution be called 2x2 Differential Transmission (2x2 DT). This dualreceiver, dual-transmitter setup can be realized with the two headlights (or taillights) and two properly positioned receivers on the back (or front) of the vehicle. Figure 1 presents this configuration with the addition of common-mode noise, affecting both receivers. In an ideal situation, the signal sent by Transmitter 1 (TX1) only reaches Receiver 1 (RX1) and TX2 RX2, respectively. As Fig. 1 shows, optical signals are sent in a complementary manner. In VLC-V2V applications, this complementary method can be considered as differential signal transmission. In the following section, the preliminary simulation results are presented for common-mode and differential-mode VLCV2V transmission.

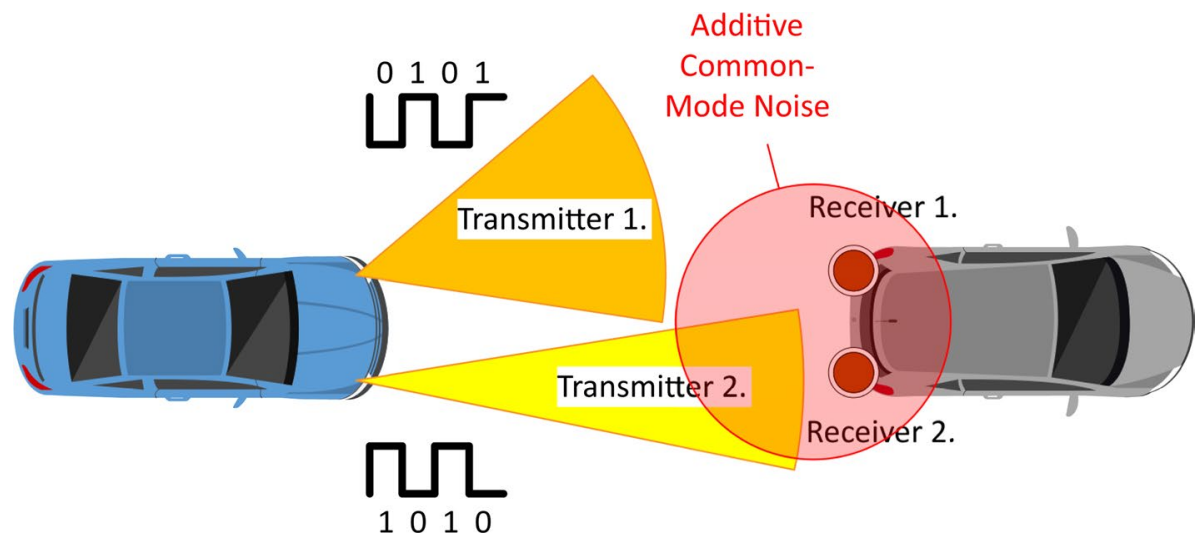

Fig. $12 \times 2$ DT concept with additive optical common-mode noise, VLC transmitters are the vehicle's headlights, receivers are attached to the rear segment of the vehicle 


\section{Mathematical background and simulation of $2 \times 2$ common-mode and $2 \times 2$ differential VLC-V2V transmission}

In order to highlight the beneficial properties of the $2 \times 2$ DT setup compared to $2 \times 2$ commonmode setup $(2 \times 2 \mathrm{CT})$, the mathematical background and simulation results are presented in this section. The mathematical background gave the base of the simulation environment implementation. The simulation environment was created in MATLAB. The presented simulation results highlight the common-mode noise reduction ability of the $2 \times 2$ DT compared to $2 \times 2 \mathrm{CT}$, via an ideal simulation setup (crosstalk and receiver noise is considered zero). The mathematical and simulation comparison of $2 \times 2 \mathrm{CT}$ and $2 \times 2 \mathrm{DT}$ provides a clear explanation, why $2 \times 2$ DT is better at special common-mode noise situations, than $2 \times 2 \mathrm{CT}$.

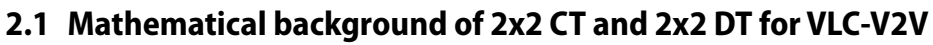

The signals on the transmitter side for the dual-transmitter setup are defined as:

$$
\underbrace{\left[\begin{array}{l}
S_{1}(t) \\
S_{2}(t)
\end{array}\right]}_{\text {TX output }}=\underbrace{\left[\begin{array}{l}
s_{1}(t) \\
s_{2}(t)
\end{array}\right]}_{\text {Source signal }}+\underbrace{\left[\begin{array}{c}
n_{\mathrm{s} 1}(t) \\
n_{\mathrm{s} 2}(t)
\end{array}\right]}_{\text {TX noise }},
$$

where $s_{1}(t)$ and $s_{2}(t)$ are the noise-free transmitter signals and $n_{\mathrm{s} 1}(t)$ and $n_{\mathrm{s} 2}(t)$ are the two additive, uncorrelated noise signals, representing internal transmitter noises.

The signals on the receiver side for the dual-receiver setup are:

$$
\underbrace{\left[\begin{array}{l}
r_{1}(t) \\
r_{2}(t)
\end{array}\right]}_{\text {RX output }}=\underbrace{\left[\begin{array}{ll}
a_{11} & a_{12} \\
a_{21} & a_{22}
\end{array}\right]}_{\begin{array}{c}
\text { Crosstalk and } \\
\text { attenuation }
\end{array}} \cdot \underbrace{\left[\begin{array}{l}
S_{1}(t) \\
S_{2}(t)
\end{array}\right]}_{\text {TX output }}+\underbrace{\sum_{j=1}^{K}\left[\begin{array}{c}
b_{\mathrm{f}, j} \cdot N^{(j)}(t) \\
\left(1-b_{\mathrm{f}, j}\right) \cdot N^{(j)}(t)
\end{array}\right]}_{\text {Common-mode noises }}+\underbrace{\left[\begin{array}{l}
n_{\mathrm{r} 1}(t) \\
n_{\mathrm{r} 2}(t)
\end{array}\right]}_{\mathrm{RX} \text { noise }}
$$

where $a_{m n}$ are constant coefficients describing the crosstalk and transmission between TXm and $\mathrm{RX} n, 0<b_{\mathrm{f}, j}<1$ is the balance factor of the $j^{\text {th }}$ common-mode noise component $N^{(j)}$, whereas $n_{\mathrm{r} 1}$ and $n_{\mathrm{r} 2}$ represent residual receiver noise components. The total number of common-mode noise components is $K$. This matrix representation contains the crosstalk between the two channels. Therefore, crosstalk can be defined as:

$$
\begin{aligned}
& X_{1 \rightarrow 2}=\frac{a_{21}}{a_{22}}: \text { crosstalk from TX1 to RX2, } \\
& X_{2 \rightarrow 1}=\frac{a_{12}}{a_{11}}: \text { crosstalk from } \mathrm{TX} 2 \text { to RX1. }
\end{aligned}
$$

The 2x2 CT method sums the two received signals $r_{1}(t)$ and $r_{2}(t)$ and the source signals $s_{1}(t)$ and $s_{2}(t)$ are equal. Therefore, the output signal for the $2 \times 2$ CT method $o^{c}(t)$ is: 


$$
\begin{aligned}
o^{c}(t)= & r_{1}(t)+r_{2}(t) \\
= & \left(a_{11}+a_{12}+a_{21}+a_{22}\right) \cdot s_{1}(t) \\
& +\left(a_{11}+a_{21}\right) \cdot n_{\mathrm{s} 1}(t)+\left(a_{12}+a_{22}\right) \cdot n_{\mathrm{s} 2}(t) \\
& +\sum_{j=1}^{K} N^{(j)}(t)+n_{\mathrm{r} 1}(t)+n_{\mathrm{r} 2}(t) .
\end{aligned}
$$

The 2x2 DT method subtracts the two received signals $r_{1}(t)$ and $r_{2}(t)$ and the source signals $s_{1}(t)$ and $s_{2}(t)$ are complementary $\left(s_{1}(t)=-s_{2}(t)\right)$. Therefore, the output signal for the $2 \times 2$ DT method $o^{d}(t)$ is:

$$
\begin{aligned}
o^{d}(t)= & r_{1}(t)-r_{2}(t) \\
= & \left(a_{11}+a_{22}-a_{12}-a_{21}\right) \cdot s_{1}(t) \\
& +\left(a_{11}-a_{21}\right) \cdot n_{\mathrm{s} 1}(t)+\left(a_{12}-a_{22}\right) \cdot n_{\mathrm{s} 2}(t) \\
& +\sum_{j=1}^{K}\left(2 \cdot b_{\mathrm{f}, j}-1\right) \cdot N^{(j)}(t)+n_{\mathrm{r} 1}(t)-n_{\mathrm{r} 2}(t) .
\end{aligned}
$$

Equation (6) clearly shows that crosstalk $\left(a_{12}\right.$ and $\left.a_{21}\right)$ influences the output signal differently. In $2 \times 2$ CT it increases the received utile signal amplitude, contrary to $2 \times 2$ DT. The aforementioned equations also imply that $2 \times 2$ DT is capable of common-mode noise reduction if $0<b_{\mathrm{f}, j}<1.2 \times 2$ DT is also capable of reducing TX noise $\left(n_{\mathrm{s} 1}\right.$ and $\left.n_{\mathrm{s} 2}\right)$, if crosstalk is not zero. However, neither $2 \times 2$ CT nor $2 \times 2$ DT is capable of reducing the independent, uncorrelated RX noises. Assuming an ideal setup, where all common-mode noise components are perfectly balanced $\left(b_{\mathrm{f}, j}=0.5 \forall j\right)$, crosstalk and receiver noises are negligible $\left(a_{12}=a_{21} \approx 0, n_{\mathrm{r} 2}=n_{\mathrm{r} 1} \approx 0\right)$ :

$$
\begin{gathered}
o^{c}(t)=\left(a_{11}+a_{22}\right) \cdot s_{1}(t)+a_{11} \cdot n_{\mathrm{s} 1}(t)+a_{22} \cdot n_{\mathrm{s} 2}(t)+\sum_{j=1}^{K} N^{(j)}(t), \\
o^{d}(t)=\left(a_{11}+a_{22}\right) \cdot s_{1}(t)+a_{11} \cdot n_{\mathrm{s} 1}(t)-a_{22} \cdot n_{\mathrm{s} 2}(t) .
\end{gathered}
$$

It is clear from Eqs. (7) and (8) that the $2 \times 2$ DT solution completely eliminates commonmode noise. The $2 \times 2$ DT equation states that transmitter noise components' reduction depends on the correlation between $n_{\mathrm{s} 1}(t)$ and $n_{\mathrm{s} 2}(t)$. If correlation is low, neither $2 \times 2 \mathrm{DT}$, nor $2 \times 2 \mathrm{CT}$ is able to reduce noise. On the other hand if correlation is high, $2 \times 2$ DT provides better noise reduction.

\subsection{Simulation comparison of $2 \times 2$ CT and $2 \times 2$ DT}

The simulation environment was implemented in MATLAB based on the equations from the mathematical background section. The aim of the simulation was to highlight the effects of added external optical noises and gain an insight into their cancellation using our proposed design. The simulation parameters are listed below: 


$$
\begin{aligned}
a_{11} & =0.2, \\
a_{22} & =0.2, \\
a_{12} & =a_{21}=0, \\
n_{\mathrm{s} 1}(t) & =\operatorname{AWGN}(\mu=0, \sigma=0.1), \\
n_{\mathrm{s} 2}(t) & =\operatorname{AWGN}(\mu=0, \sigma=0.1), \\
N^{(1)}(t) & =\operatorname{Square}(A=0.1, f=10 \mathrm{kHz}), \\
N^{(2)}(t) & =\operatorname{Square}(A=0.1, f=57.6 \mathrm{kHz}),
\end{aligned}
$$

where AWGN $(\mu, \sigma)$ is an Additive White Gaussian Noise with expected value $\mu$ and standard deviation $\sigma$ and $\operatorname{Square}(A, f)$ is a symmetrical square signal with half-amplitude $A$ and frequency $f$.

In the $2 \times 2 \mathrm{CT}$ setup, the utile signals were: $s_{1}(t)=\operatorname{PRND}(0.1,115.2 \mathrm{kbps})$ and $s_{2}(t)=s_{1}(t)$, whereas in $2 \times 2$ DT: $s_{1}(t)=\operatorname{PRND}(0.1,115.2 \mathrm{kbps})$ and $s_{2}(t)=-s_{1}(t)$. $\operatorname{PRND}(B, R)$ is a pseudo-random bitsequence function that generates a symmetrical OOK signal, with half-amplitude $B$ and bitrate $R$. All signal amplitudes are in arbitrary units. The bitrate value was chosen, as it's the highest available UART bitrate that is supported by a wide-range of serial transmitter devices. For better visibility, the number of randomly generated transmitted bits was limited to 40. The simulated transmission is affected by two balanced common-mode square signals $N^{(1)}(t)$ and $N^{(2)}(t)$, which equally affect both receivers. These noise signals are denoted as Noise 1, Noise 2 in Figs. 2 and 3.

An additional non-common-mode AWGN source was included in order to highlight the effect of uncorrelated (non-common-mode) noise types. Other optical and electrical noise components, like receiver shot noise, thermal noise, etc. were omitted in simulation, as their influence was considered to be much lower, than the added noise components.

Evaluation of the comparison results In Fig. 2, the $2 \times 2$ CT setup is demonstrated: the two RX signals are added together, the output signal is the sum of RX1 and RX2. The upper segments show the transmitted signal (TX1 and TX2) and the superimposed noise signals (Noise 1 and Noise 2). The lower segment presents the received signals (RX1 and RX2) with green color. The lower left segment of Fig. 2 shows the signal after the sum operation (Output Signal). However, common-mode noise is entirely present in the output signal, resulting a low Q-factor (2.58) and high BER $\left(4.9 \cdot 10^{-3}\right)$. The amplitude distribution diagram shows the effects of common-mode noise, the distribution is spread out. Therefore bit error probabilities are higher. The difference between this solution and $2 \times 2$ DT transmission is easy to highlight.

Fig. 3 presents the results for the proposed $2 \times 2$ DT setup. The upper segments show the transmitted signal (TX1 and TX2) and the superimposed noise signals (Noise 1 and Noise 2). The lower segment presents the received signals (RX1 and RX2) with green color. The lower left figure segment shows the signal after the subtraction operation (Output Signal). Lastly, the lower right segment shows the amplitude distribution of the output signal for Q-factor and Bit Error Rate (BER) calculation purposes. The output signal in this setup is entirely free from common-mode noise and has double the amplitude of each TX signal. In this arrangement, common-mode noise is efficiently filtered out, and the output signal amplitude is doubled, as expected. This resulted in a substantially high Q-factor (5.69) and a BER value of $6.09 \cdot 10^{-9}$. It is important to mention that the AWGN noise is resistant to the subtraction opreation, hence it affects both the $2 \times 2 \mathrm{CT}$ and $2 \times 2$ DT similarly. Based on these promising results, it can be safely stated that the $2 \times 2$ DT setup may be suitable for common-mode noise rejection in VLC-V2V 

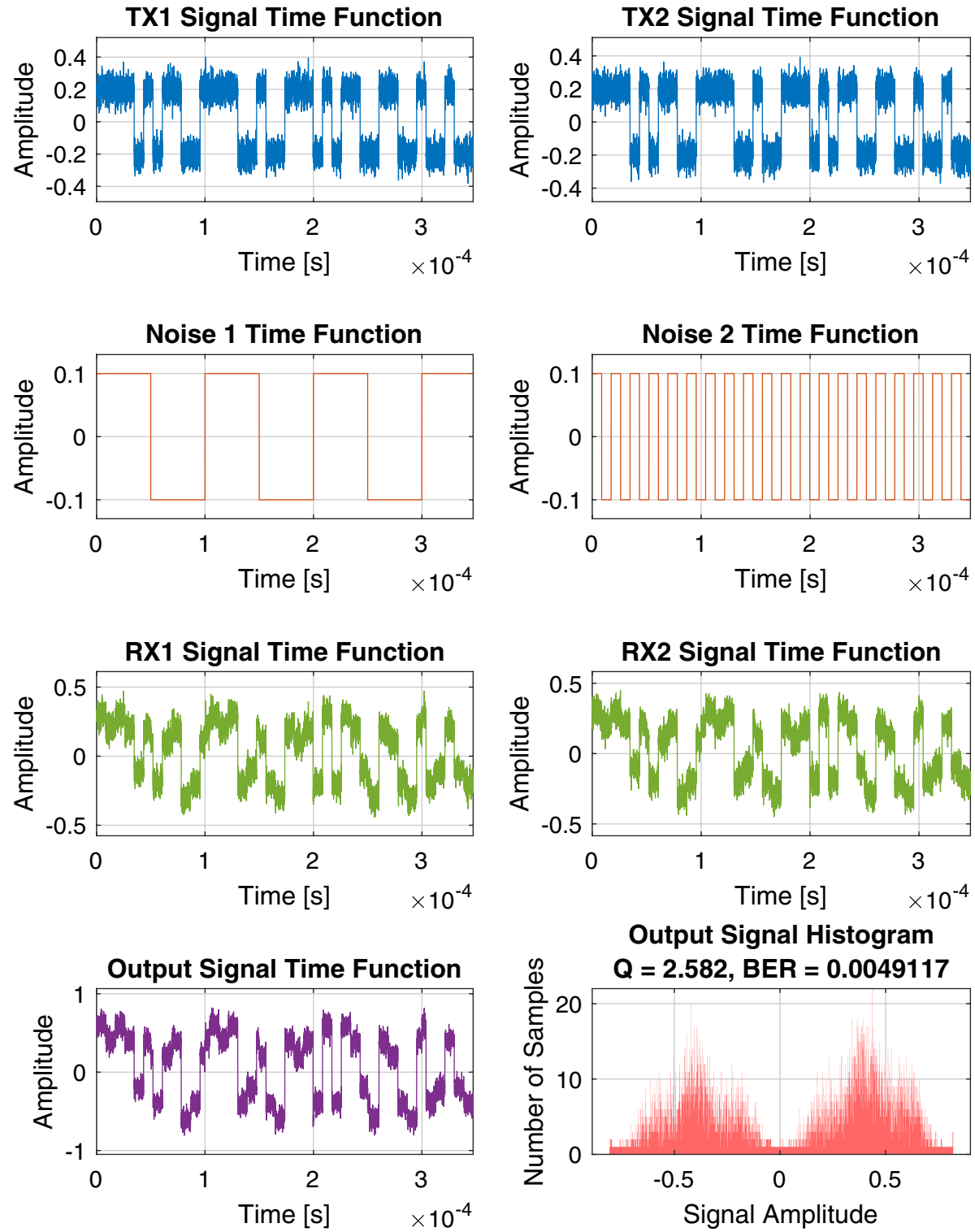

Fig. 2 2x2 CT simulation results. Amplitude values are in arbitrary units, time scale is in seconds. The two transmitter signal functions (TX1, TX2) are influenced by two balanced common-mode noises (Noise 1 and Noise 2). The effects of common-mode noise are perceivable in the received signal (RX1 and RX2). The output signal in this case is the sum of RX1 and RX2. The signal amplitude distribution is spread out, resulting in weak performance and high BER

applications. Provided that there is a low amount of crosstalk between the two transmission routes and the majority of channel noises are identified as common-mode noise, this method will perform better compared to $2 \times 2 \mathrm{CT}$. In the following section, the identification results of VLC-V2V channel noise sources are presented under low-light, 

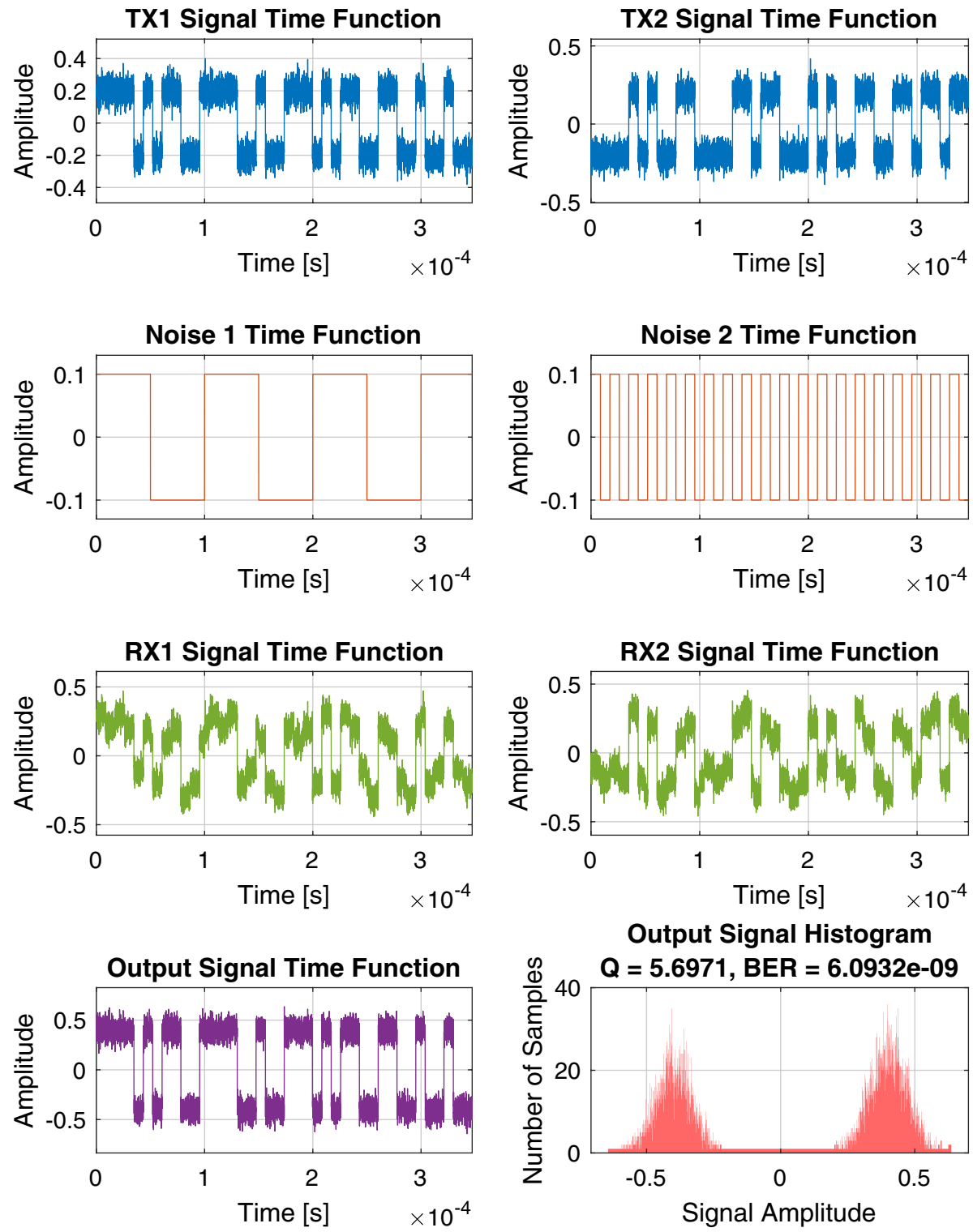

Fig. $32 \times 2$ DT simulation results. Amplitude values are in arbitrary units, time scale is in seconds. The two transmitter signal functions (TX1, TX2) are influenced by two balanced common-mode noises (Noise 1 and Noise 2). The effects of common-mode noise are percieveable in the received signal (RX1 and RX2). The output signal in this case is the difference of RX1 and RX2. The signal amplitude distribution is narrower, resulting in better performance and lower BER 
urban road conditions. The effects of crosstalk between propagation paths is addressed later.

\section{Noise identification and rejection performance using the $2 \times 2$ receiver structure}

Our first goal is to identify the nature of artificial noise sources that are present in the VLC-V2V channel under low-light environmental conditions. By using a 2x2 differential receiver structure, the best noise rejection performance is available when both receivers are affected by balanced common-mode noise. This type of noise may originate from public road lighting (shown in Fig. 4). A dedicated measurement setup was designed for investigating whether this type of noise rejection technique performs well in a real-world road test.

Measurement setup and devices First, the two photodiode-based amplified detectors were attached to the bottom segment of the car's trunk lid (denoted with red dots in Fig. 4 and pointed at with yellow arrows in Fig. 7). The two receivers were horizontally $80 \mathrm{~cm}$ apart. The signal of both detectors and the differential amplifier, which subtracts the two detector signals were fed into a data acquisition unit (DAQ): RedLab 204 USB Mini DAQ. The DAQ was placed on the backseat of the vehicle and was connected to a portable PC via USB. The measurement setup's block diagram is shown in Fig. 6. These signals were recorded with a sample rate of $100 \mathrm{kSample} / \mathrm{s}$. The measurement took place in an urban area, under low-light conditions at night, on roads with public lighting. The measurement

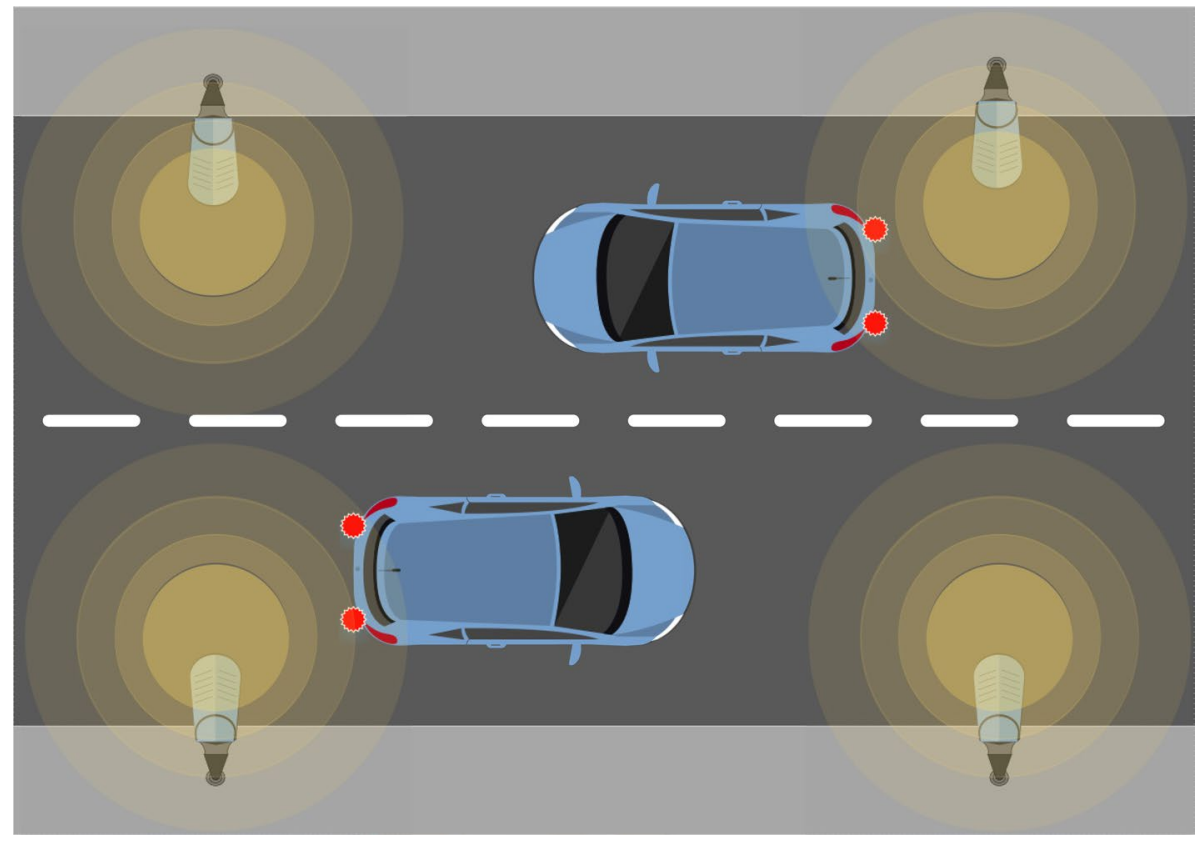

Fig. 4 Effects of public lighting on VLC-V2V channel, VLC receivers denoted with red 
lasted for 600 seconds altogether. The vehicle moved along the route, obeying the speed limits and laws of the road with an average speed of approximately $25 \mathrm{~km} / \mathrm{h}$.

Measurement data post-processing The data collected throughout the measurement was post-processed in a MATLAB environment. For the demonstration of common-mode noise rejection performance, the discrete Fourier transforms (using the built-in FFT algorithm of MATLAB) was used instead of the recorded time-domain signals. As there were approximately 60 million independent time-domain samples from each signal (RX1, RX2, Differential Output), it would not be easy to visualize the noise rejection performance using time-domain signals. Fig. 5 shows a portion of the spectral distribution of the three signals (RX1-black, RX2-yellow, Differential Output-red). As we did not encounter significant higher frequency components above $6.5 \mathrm{kHz}$, the inset shows components up to $6.5 \mathrm{kHz}$. The original plot in Fig. 5 highlights the dominant measured frequency components up to $1 \mathrm{kHz}$.

Evaluation of measurement results Based on work by Fekete et al. in Fekete (Aug. 2015), the public lighting alongside the measurement route is assume-ably based on incandescent lamps, as the odd and even harmonics of the $50 \mathrm{~Hz}$ driving frequency is present. Interestingly the harmonics of $100 \mathrm{~Hz}$ are even more dominant in our measurement. Fig. 5 shows the sum and the difference signal spectra of receiver RX1 and RX2. The benefit of the difference signal is clearly present, as its peaks (red) are significantly lower than the summed signal spectra (orange). As the proposed system's measured common-mode noise reduction performance is promising, we can safely state that the investigated VLC-V2V channel contains a significant

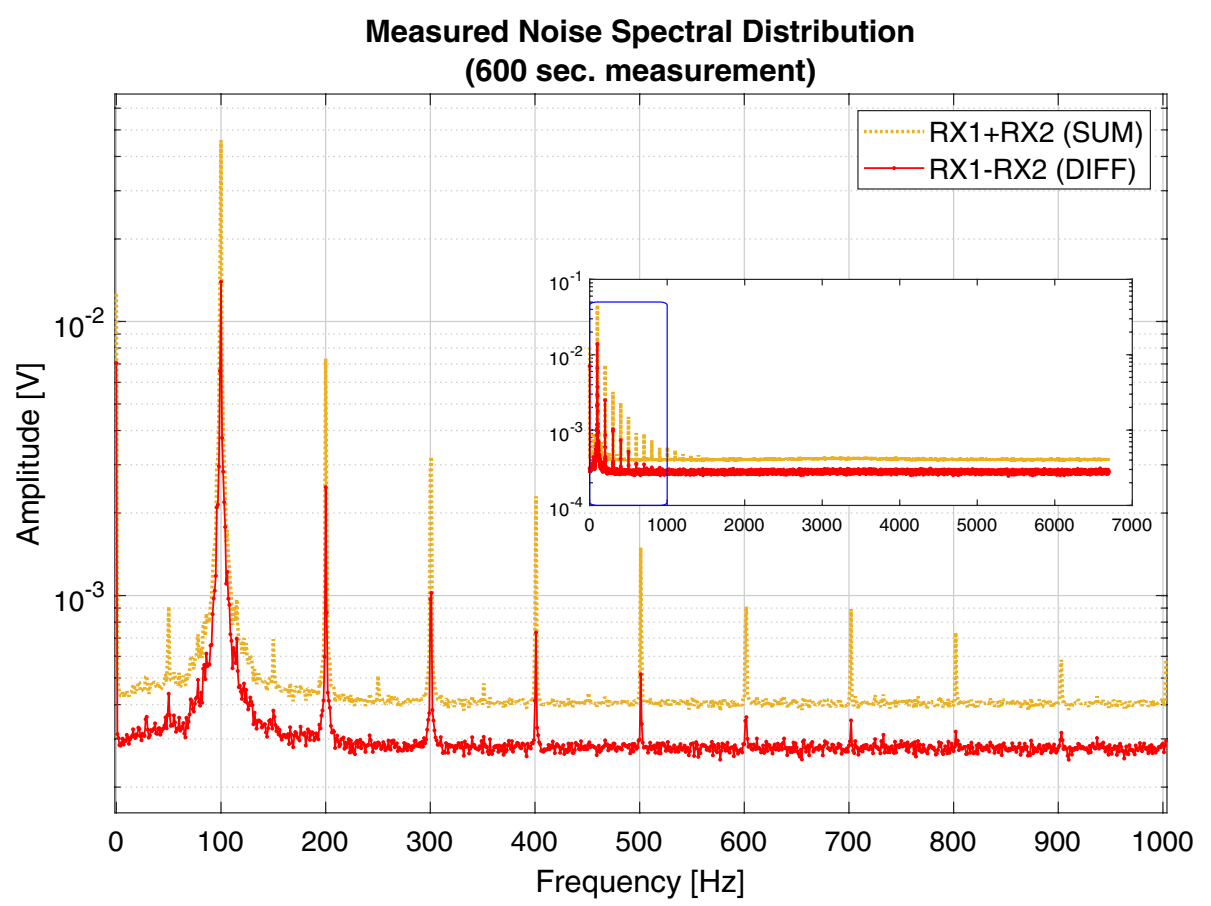

Fig. 5 Normalized noise spectral distribution of the $600 \mathrm{~s}$ road test measurement. The red function represents the output of the differential amplifier, orange function is the sum of RX1 and RX2. The noise reduction is clearly present, the differential receiver reduced common-mode noises significantly 
Table 2 Peak noise amplitude reduction performance with the differential receiver setup. The values were calculated from the peak values of Fig. 5

\begin{tabular}{llllll}
\hline Frequency & $100 \mathrm{~Hz}$ & $200 \mathrm{~Hz}$ & $300 \mathrm{~Hz}$ & $400 \mathrm{~Hz}$ & $500 \mathrm{~Hz}$ \\
\hline Peak reduction & $43.8 \%$ & $50.1 \%$ & $45.6 \%$ & $46.8 \%$ & $52.77 \%$ \\
\hline
\end{tabular}

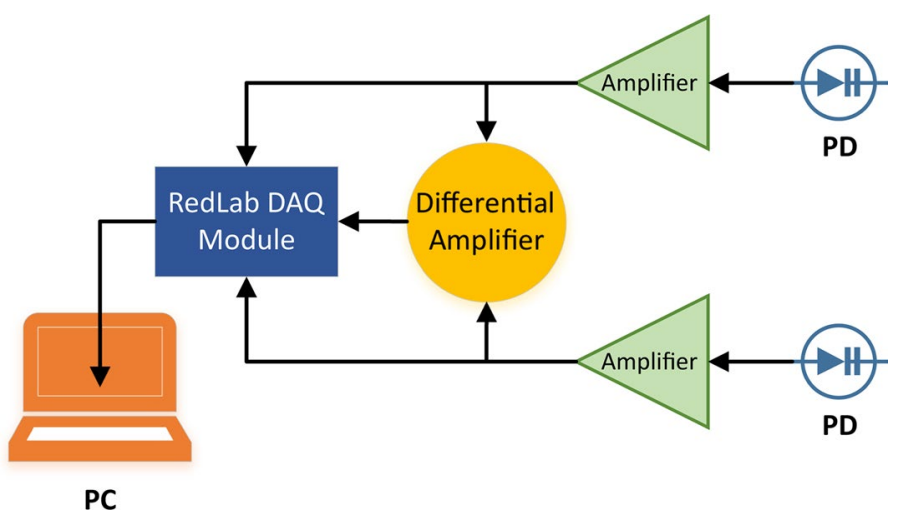

Fig. 6 Road test measurement setup block diagram

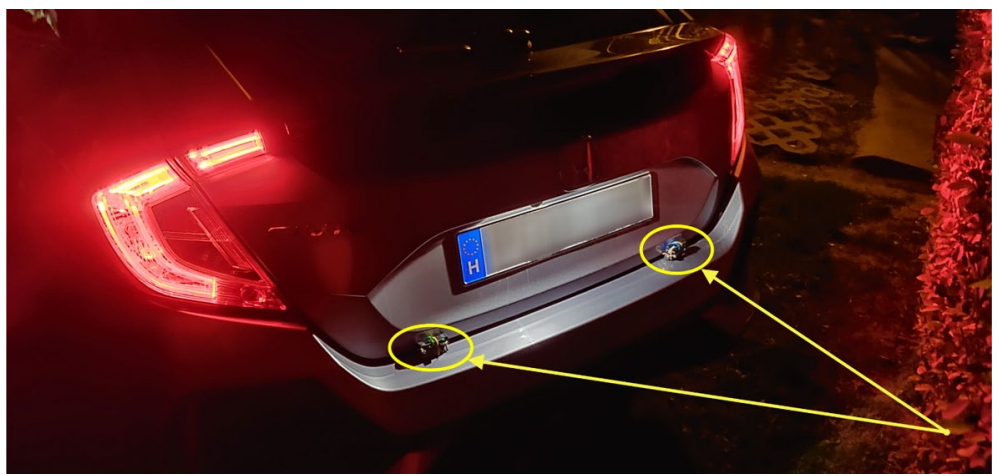

Fig. 7 Receiver arrangement on the car's trunk lid. The receiver's signal harness was fed through underneath the trunk lid seal. The data acquisition device and the PC was located inside the trunk

amount of common-mode noise, caused by public lighting at night. Our proposed method (differential receiver) for reducing this noise type performed successfully in the real-life road test Table 2. 


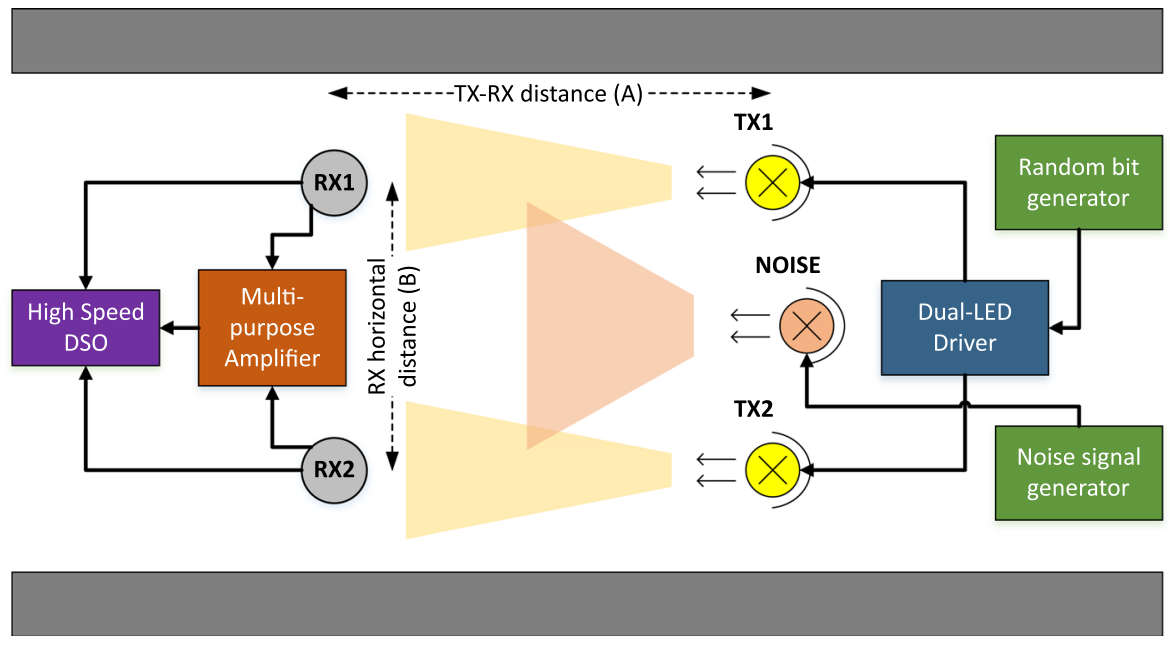

Fig. 8 The setup for $2 \times 2$ transmission comparison measurements

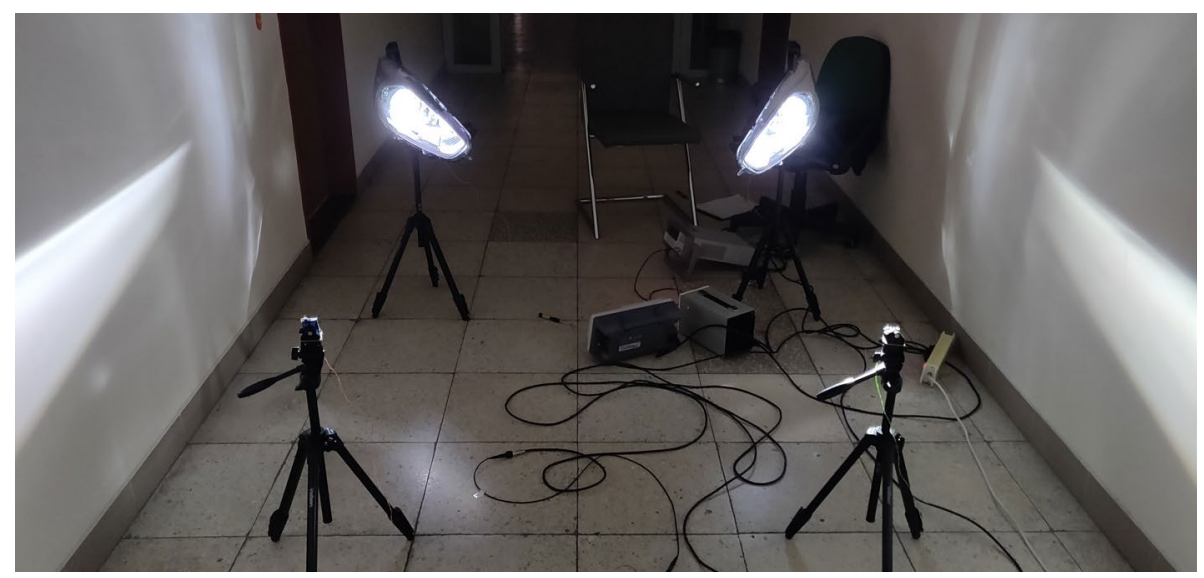

Fig. 9 Photograph of the setup for $2 \times 2$ transmission measurements without additional noise source

\section{VLC-V2V transmission measurements by automotive grade headlights}

For demonstrational purposes we've built an indoor VLC-V2V transceiver setup. The schematic diagram is shown in Fig. 8 and the measurement setup in Figs. 9 and 10. The arrangement contained two automotive-grade headlights and two custom-built photodiode based amplified detectors. The TX-RX distance (parameter A in Fig. 8) was gradually adjusted from $2 \mathrm{~m}$ up to $10 \mathrm{~m}$. The RX horizontal distance (parameter B in Fig. 8) was set to $1.2 \mathrm{~m}$. The link quality is graded by the Q-factor of the receiver signals.

Transmitter side (TX) The headlight housings contained a $15 \mathrm{~W}$ H7-socket type LED light each (with the built-in driving circuit removed). Each headlight was attached to a 


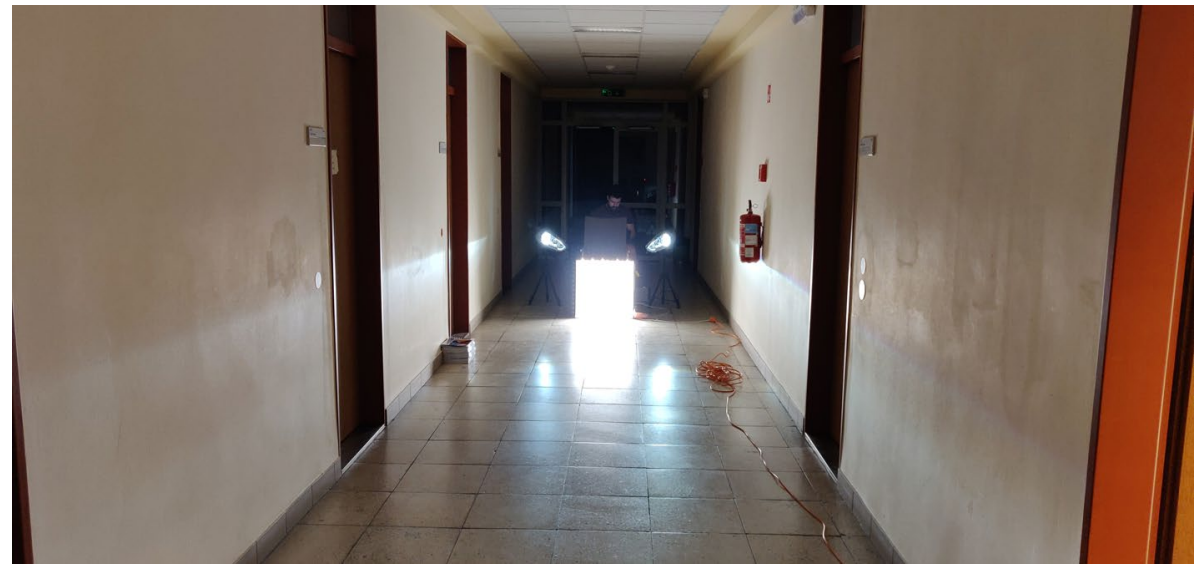

Fig. 10 Photograph of the setup for $2 \times 2$ transmission measurements with activated noise source

customized VLC driver circuit capable of differential (2x2 DT) and common-mode $(2 \times 2$ CT) transmission. The transmitted signal in our setup was a pseudo-random bitstream with a data rate of $115.2 \mathrm{kbps}$ (square signal, OOK modulation).

Receiver side $(R X)$ The two receivers were attached to adjustable height tripods for accurate positioning. The receiver signals were fed to a multi-purpose amplifier (able to operate differentially or in common-mode). Both the receiver signals and the multi-purpose amplifier's signal was analyzed with a High-Speed Digital Storage Oscilloscope (HS-DSO).

Noise source (NS) For noise rejection performance evaluation purposes, a high-power $100 \mathrm{~W}$ LED-based noise source was added to the setup. This noise source was precisely positioned in such a way that it affects both receivers with the same amount of noise. This is a common-mode noise source in our setup. The driving signal for this LED is an independent pseudo-random bitstream with the same $115.2 \mathrm{kbps}$ data rate and OOK modulation. In this scenario, this can be interpreted as a worst-case common-mode in-band noise. The noise source can be toggled on and off between measurements.

\subsection{Measurement comparison of $2 \times 2 \mathrm{CT}$ and $2 \times 2 \mathrm{DT}$ common-mode noise reduction performance}

Our goal with this measurement was to show that our suggested 2x2 DT VLC-V2V arrangement performs better than the conventional $2 \times 2$ CT solution. We set up 11 measurement distances, equidistantly spaced between 2 and 10 meters and measured the transmission quality with and without the presence of common-mode noise. Activated and deactivated noise source setups are shown in Figs. 9 and 10. Eye-diagrams and the corresponding Q-factors were obtained from the HS-DSO. The approximate BER values were calculated from the Q-factor values for more exact comparison. The following two formula was used for the BER approximation:

$$
\mathrm{BER} \approx \frac{1}{2} \operatorname{erfc}\left(\frac{Q}{\sqrt{2 \pi}}\right),
$$

where erfc stands for the Gaussian complementary error function. 


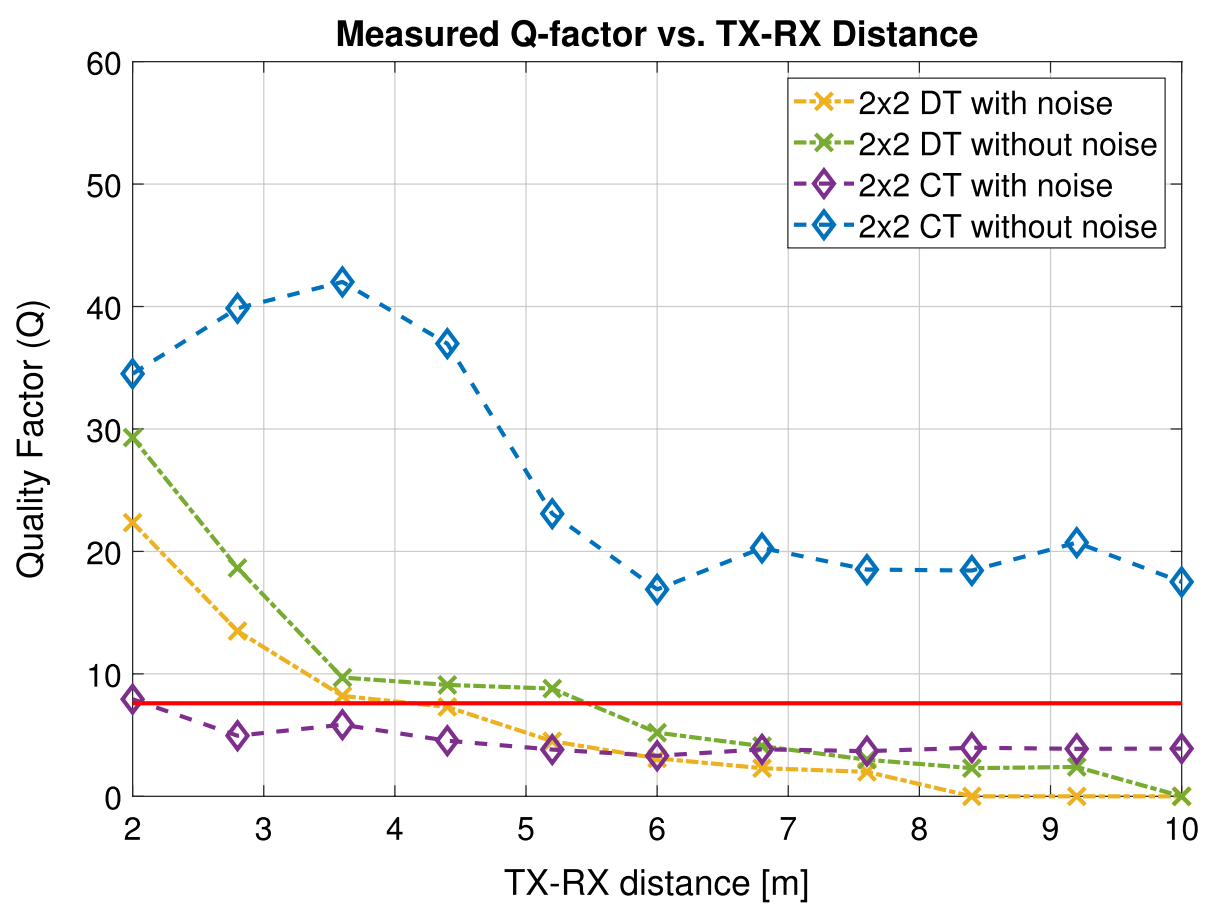

Fig. 11 Measured Q-factor vs. TX-RX Distance

\subsubsection{Measurement results}

The $2 \times 2$ measurement results are shown in Fig. 11. The best transmission performance was available with the $2 \times 2 \mathrm{CT}$ arrangement when the noise source was deactivated (denoted in blue with dashed line). The lowest acceptable Q-factor was 7.6, which is approximately equal to a BER value of $1 \cdot 10^{-14}$. This Q-factor limit is denoted with a solid red line on Fig. 11. In practice, if the VLC-V2V channel's common-mode noise is low (or zero), the best transmission performance is available with the $2 \times 2 \mathrm{CT}$ arrangement. However, if balanced common-mode noise is significant, transmission performance dramatically degrades. Therefore, in these situations, the $2 \times 2 \mathrm{CT}$ arrangement exhibited weak performance. The difference between $2 \times 2 \mathrm{CT}$ and $2 \times 2 \mathrm{DT}$ is clear. In case of $2 \times 2$ DT, the balanced common-mode noise did not influence transmission quality as noticeably, as in $2 \times 2 \mathrm{CT}$ arrangement. For distances between 2 and 6 meters, $2 \times 2$ DT (shown in green and yellow color in Fig. 11) outperformed the $2 \times 2 \mathrm{CT}$ arrangement in presence of balanced common-mode noise. On the other hand, for distances above 6 meters, 2x2 DT transmission had lower performance in every setup. Our assumption for this phenomenon was that it's ought to be due to the raised amount of crosstalk between channels. The crosstalk directly degraded transmission performance, as previously presented in the Sect. 2. In this arrangement, crosstalk mainly originated from the divergence of the light beams emitted by the two LED headlights. Crosstalk reduction is addressed later in Sect. 4.2. 


\subsubsection{Measurement conclusion}

The results proved that the $2 \times 2 \mathrm{DT}$ is a promising candidate for balanced common-mode noise rejection. $2 \times 2$ DT performed better than $2 \times 2 \mathrm{CT}$ in balanced common-mode noise situations (where the TX-RX distance was less than 6 meters). Above TX-RX distances of 6 meters, the $2 \times 2$ DT performed weaker than $2 \times 2 \mathrm{CT}$, due to the presumably more significant amount of crosstalk (overlap) between light beams at large distances. For distances less than 6 meters and environments where common-mode noise is present, $2 \times 2$ DT performed better. In noise-free situations, the best performance was available with the $2 \times 2 \mathrm{CT}$ arrangement. After these results, our goal was to enhance the 2x2 DT setup's performance by reducing the crosstalk.

\subsection{Crosstalk reduction for $2 \times 2$ DT with field-of-view (FOV) limiters on receivers}

The crosstalk between the two propagation routes can either be reduced by changing the divergence and altering the projected image of the headlights or changing the receivers' spatial sensitivity characteristics. In VLC-V2V communication, both solutions are available:

- electrically controlled headlight projection image using segmented LED arrays

- application of a Field-of-View (FOV) limiter to the amplified detector

In 2x2 DT transmission the crosstalk value plays a key role, as high crosstalk eliminates the utile signal together with common-mode noise (see Sect. 2). This leads to higher BER values and lower Q-factor. Balanced common-mode noise at the receivers and zero crosstalk between transmitters and receivers provides the best result for $2 \times 2$ DT transmission. However, for some cases where significant crosstalk between channels is inevitable, $2 \times 2$ CT transmission might be the better option, especially if common-mode noise amplitudes are significantly lower than the utile signal. In order to reduce crosstalk in our system, the FOV limiter option was chosen, due to our transmitter headlight limitations and the more frequent use of H7 LED-based headlights in automotive designs. Our FOV limiter attached to the amplified detector reduced the receiver FOV to 30 in both horizontal and vertical directions. This FOV limiter dramatically reduced the crosstalk between the channels, as will be shown by the results in the following section.

Measurement results with receiver FOV limiter The FOV limiter was added to both receivers, and measurements were conducted similarly to the previous section. As seen in Fig. 12, the Q-factors improved significantly in the case of 2x2 DT, compared to the previous measurement. Similarly to the previous measurement the lowest acceptable Q-factor was identically 7.6, which is approximately equal to a BER value of $1 \cdot 10^{-14}$. This Q-factor limit is denoted with a solid red line on Fig. 12. It is also important to highlight that the conventional 2x2 CT fails in presence of common-mode noise for distances above 4 meters. However, clearly due to the FOV limiter the transmission quality is better for distances less than 4 meters, compared to the previous measurement. In balanced commonmode noise situations, the $2 \times 2 \mathrm{DT}$ solidly outperformed $2 \times 2 \mathrm{CT}$, as expected. This result verified our previous assumption. The significant performance decay in $2 \times 2$ DT cases for distances higher than 6 meters in the previous measurement setup was caused by crosstalk. The receiver FOV limitation reduced crosstalk, hence the 2x2 DT quality parameters 


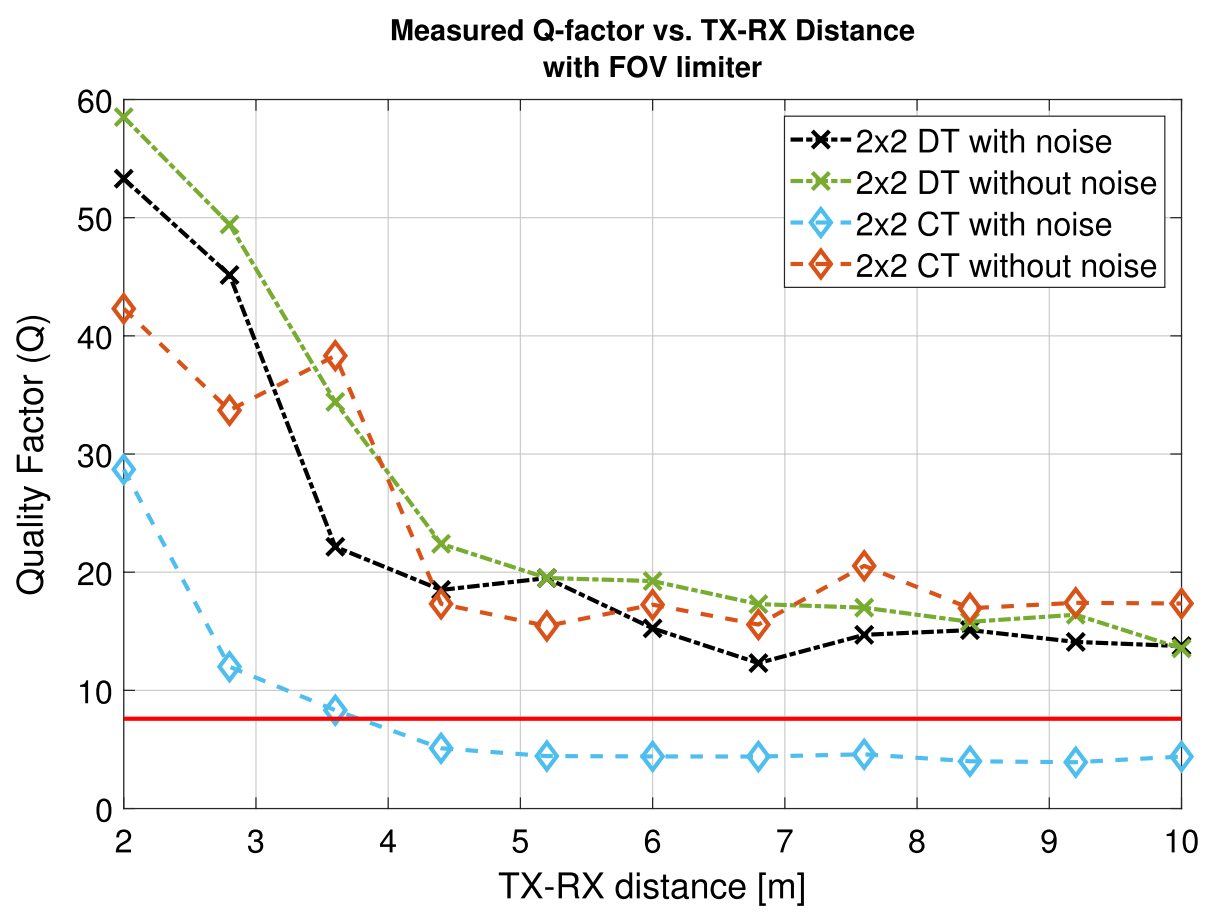

Fig. 12 Measured Q-factor vs. TX-RX Distance, with FOV limiter

significantly improved. The results clearly showed that, our proposed $2 \times 2$ DT solution resulted a higher Q-factor than our prescribed lowest limit (7.6), in all cases. Furthermore, balanced common-mode noise does not affect $2 \times 2$ DT as significantly as the $2 \times 2$ CT solution. To summarize, we can safely state that when balanced common-mode noise is present, our proposed $2 \times 2$ DT solution performs better than conventional $2 \times 2 \mathrm{CT}$.

\section{Conclusion}

In this paper we introduced an alternative VLC-V2V communication method targeting cost-efficiency and enhanced common-mode noise rejection performance. Our real-world vehicle road tests confirmed that the VLC-V2V channel contains a significant amount of common-mode noise. This originates from public lighting, and our proposed method suppressed this noise well (as seen in Sect. 3.). Later on, our system was compared to a conventional common-mode transmission system (found in several published papers) with and without the presence of balanced common-mode noise (Sect. 4.1.). We validated our $2 \times 2$ DT arrangement through simulation and measurements. The $2 \times 2$ DT arrangement performs better than $2 \times 2 \mathrm{CT}$ in the presence of balanced common-mode noise. However, for TX-RX distances above 6 meters, our 2x2 DT method failed to perform better, due to increased crosstalk between transmitter light beams. Therefore, we suggested a solution for crosstalk reduction. An additional Field-of-View (FOV) limiter on the receivers resulted in significantly lower crosstalk and better transmission quality even for distances above 6 
meters. Summarizing the above, our suggested $2 \times 2$ DT solution proved to be better than $2 \times 2 \mathrm{CT}$, in cases where common-mode noise was present. We delivered reliable $115 \mathrm{kbps}$ UART stream with BER values under $1 \cdot 10^{-14}$ for distances up to $10 \mathrm{~m}$ in presence of significant in-band, common-mode noise with our $2 \times 2$ DT design. Therefore, for such critical applications like VLC-V2V communication our 2x2 DT solution provides better reliability and noise reduction performance than conventional solutions.

Acknowledgements The research reported in this paper was supported by the BME Artificial Intelligence FIKP grant of EMMI (BME FIKP-MI/SC) and BME NC TKP2020 grant of NKFIH Hungary.

Funding Open access funding provided by Budapest University of Technology and Economics.

Open Access This article is licensed under a Creative Commons Attribution 4.0 International License, which permits use, sharing, adaptation, distribution and reproduction in any medium or format, as long as you give appropriate credit to the original author(s) and the source, provide a link to the Creative Commons licence, and indicate if changes were made. The images or other third party material in this article are included in the article's Creative Commons licence, unless indicated otherwise in a credit line to the material. If material is not included in the article's Creative Commons licence and your intended use is not permitted by statutory regulation or exceeds the permitted use, you will need to obtain permission directly from the copyright holder. To view a copy of this licence, visit http://creativecommons.org/licenses/by/4.0/.

\section{References}

Adiono, T., et al. : Analog filters design in VLC analog front-end receiver for reducing indoor ambient light noise. In: Proc. IEEE Asia Pacific Conf. Circuits and Systems (APCCAS). Oct. (2016), pp. 581-584. https://doi.org/10.1109/APCCAS.2016.7804058

Ahmed, M., Bermak, A.: A CMOS Transimpedence Amplifier with am- bient light rejection for visible light communication in intelligent trans- port systems. In: Proc. 15th Int. wireless communications mobile com- puting Conf. (IWCMC). June (2019), pp. 7-13. https://doi.org/10.1109/IWCMC.20198766535

Alam, M. R., Faruque, S.: Prospects of differential optical receiver with ambient light compensation in vehicular visible light communication. In: Proc. IEEE Vehicular Networking Conf. (VNC). (2016), pp. 1-4

Alsalami, F. M., et al. "Regular-shaped geometry-based stochastic model for vehicle-to-vehicle visible light communication channel". In: Proc. IEEE Jordan Int. Joint Conf. Electrical Engineering and Information Technology (JEEIT). (2019), pp. 297-301

Beshr, M., Michie, C., Andonovic,v: Evaluation of visible light com- munication system performance in the presence of sunlight irradiance'. In: Proc. 17th Int. Conf. Transparent Optical Networks (ICTON). July (2015), pp. 1-4. https://doi.org/10.1109/ICTON.2015.7193685.

Chang, F., et al. :Design and implementation of anti low-frequency noise in visible light communications. In: Proc. Int. Conf. Applied System Innova- tion (ICASI). May (2017), pp. 1536-1538. https://doi.org/ 10.1109/ICASI.2017.7988219.

Chen, A., et al. "Time variation in vehicle-to-vehicle visible light communi- cation channels". In: Proc. IEEE Vehicular Networking Conf. (VNC). (2016), pp. 1-8

Cheng, L., et al.: Comparison of radio frequency and visible light propa- gation channels for vehicular communications. IEEE Access 6, 2634-2644 (2018)

Chung,Y. H., Oh,S.:Efficient optical filtering for outdoor visible light communications in the presence of sunlight or articifical light. In: Proc. Int. Symp. Intelligent Signal Processing and Communication Systems. Nov. (2013), pp. 749-752. https://doi.org/10.1109/ISPACS.2013.6704649.

Elamassie, M., et al. "Effect of fog and rain on the performance of vehicular visible light communications". In: Proc. IEEE 87th Vehicular Technology Conf. (VTC Spring). (2018), pp. 1-6

Eldeeb, H. B., Miramirkhani, F., Uysal,M.: "A path loss model for vehicle-to-vehicle visible light communications". In: Proc. 15th Int. Conf. Telecommunications (ConTEL). July (2019), pp. 1-5. https://doi.org/ 10.1109/ConTEL.2019.8848562

Eso, E., et al. "Experimental investigation of the effects of fog on optical camera-based VLC for a vehicular environment". In: Proc. 15th Int. Conf. Telecommunications (ConTEL). (2019), pp. 1-5 
Udvary, E. "Visible light communication survey". In: Infocommunications Journal 11.2, pp. 22-31. issn: 2061-2079 (June 2019)

Fekete, G., et al.: Visible light communication channel disturbances and examination of the modulation formats. Int. J. Microw. Wirel. Technol. 8(8), 1163-1171 (2015). https://doi.org/10.1017/s175907871 5001269

Ferraz, PAP., Santos,I. S: "Visible light communication applied on vehicle-to-vehicle networks". In: Proc. Electronics and Automotive Engi- neering (ICMEAE) 2015 Int. Conf. Mechatronics. (2015), pp. 231-235

Halawi, S., et al.: Performance analysis of circular color shift keying in VLC systems with camera-based receivers. IEEE Transactions Commun. 67(6), 4252-4266 (2019)

Islim, M. S., et al. "The impact of solar irradiance on visible light communi- cations. J. Lightwave Technol. 36.12 (June) (2018), pp. 2376- 2386. issn: 1558-2213. https://doi.org/10.1109/JLT.2018.2813396.

Jong-Ho, Y., et al. "Demonstration of vehicular visible light communication based on LED headlamp". In: Proc. Fifth Int. Conf. Ubiquitous and Future Networks (ICUFN). (2013), pp. 465-467

Karbalayghareh, M., et al. "Channel modelling and performance limits of vehicular visible light communication systems". In: IEEE Transactions on Vehicular Technology (2020), p. 1

Kim, J., et al. "Vehicle-to-vehicle visible light communications using sub-pulse Manchester modulation". In: Proc. Sixth Int. Conf. Ubiquitous and Future Networks (ICUFN). (2014), pp. 481-482

Kim, Y.H., Cahyadi, W.A., Chung, Y.H.: Experimental demonstration of VLC-based vehicle-to-vehicle communications under fog conditions. IEEE Photonics J. 7(6), 1-9 (2015)

Lee, S. J., et al. "Simulation modeling of visible light communication chan- nel for automotive applications". In: Proc. 15th Int. IEEE Conf. Intelligent Transportation Systems. (2012), pp. 463-468

Mohammed, D., et al. "Digital data transmission via Visible Light Commu- nication (VLC): Application to vehicle to vehicle communication”. In: Proc. 4th Int. Conf. Control Engineering Information Technology (CEIT). (2016), pp. 1-5

Shen, W., Tsai, H.: Testing vehicle-to-vehicle visible light communica- tions in real-world driving scenarios. In: Proc. IEEE Vehicular Networking Conf. (VNC). (2017), pp. 187-194

Siddiqi, K., Raza, A. D., Muhammad,S. S.: "Visible light communica- tion for V2V intelligent transport system". In: Proc. Int. Conf. Broadband communications for next generation networks and multimedia applications (CoBCom). (2016), pp. 1-4

Singh, G., Srivastava, A., Bohara, V. A.: Impact of Weather Condi- tions and Interference on the Performance of VLC based V2V Communi- cation. In: Proc. 21st Int. Conf. Transparent Optical Networks (ICTON). (2019), pp. 1-4

Takai, I., et al:: Optical vehicle-to-vehicle communication system using LED transmitter and camera receiver. IEEE Photonics J. 6(5), 1-14 (2014)

Tebruegge,C., Memedi,A., Dressler,F.: Empirical Characterization of the NLOS Component for Vehicular Visible Light Communication. In: Proc. IEEE Vehicular Networking Conf. (VNC). (2019), pp. 1-4

Tebruegge,C., Zhang,Q., Dressler,F.: Optical interference reduction with spatial filtering receiver for vehicular visible light communication. In: Proc. IEEE Intelligent Transportation Systems Conf. (ITSC). Oct. (2019), pp. 3055-3061. https://doi.org/10.1109/ITSC.2019.8917042.

Uysal, M., et al.: Visible light communication for vehicular networking: per-formance study of a V2V system using a measured headlamp beam pattern model. IEEE Vehicular Technol Mag 10(4), 45-53 (2015)

Publisher's Note Springer Nature remains neutral with regard to jurisdictional claims in published maps and institutional affiliations. 